\title{
Hemidiaphragmatic paralysis with continuous interscalene block and diluted solutions of levobupivacaine
}

\section{Bloqueo interescalénico y parálisis diafragmática con dosis diluidas de levobupivacaína}

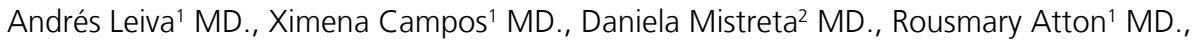
Diego Villegas ${ }^{1}$ MD., Marcos Guerrero ${ }^{1}$ MD., Aldo Campusano ${ }^{1}$ MD., Eduardo Rajdl ${ }^{1}$ MD., Andrea Fernández ${ }^{1}$ UN., Julián Aliste ${ }^{1,3}$ MD.

\begin{abstract}
The continuous interscalene block represents the analgesic standard for shoulder surgery. However, the incidence of hemidiaphragmatic paralysis can reach up to $100 \%$ of cases. We hypothesized that more dilute local anesthetics would decrease the phrenic palsy at 24 hours. Methods: Prospective series of patients undergoing arthroscopic shoulder surgery with continuous interscalene block. A $15-\mathrm{ml}$ bolus of lidocaine $1 \%$-levobupivacaine $0.25 \%$ plus an infusion of levobupivacaine $0.04 \%$ at an $8 \mathrm{~mL} / \mathrm{h}$ rate plus $5 \mathrm{~mL}$ boluses on-demand with a 20-minutes lockout was used until discharge. Hemidiaphragmatic excursion was evaluated with M-mode ultrasound in the subcostal region before blocks, in the post-anesthetic unit, and at $24 \mathrm{~h}$. The primary outcome was the presence of hemidiaphragmatic paralysis at 24 hours. Secondary outcomes included postoperative pain, amount of rescue boluses, postoperative opioids consumption, and side effects. Results: Thirty patients were recruited and analyzed. The incidence of diaphragm paralysis at $24 \mathrm{~h}$ was $96.7 \%$. The median [IQR] of pain at rest (patients with shoulder immobilizer) in a NRS from 0 to 10 at $0.5 ; 1 ; 3 ; 6 ; 12 ; 24 ; 48 ; 72$ hours were 0 [0-0]; 0 [0-0]; 0 [0-0]; 0 [0-0]; 0
\end{abstract}

\section{Key words:}

Continuous interscalene block, hemidiaphragmatic paralysis, shoulder surgery, MeSH terms: brachial plexus block

Department of Anesthesiology, Clínica Las Condes. Santiago, Chile.

Department of Anesthesiology, Hospital Luis Calvo Mackenna. Santiago, Chile.

Department of Anesthesiology and Perioperative Medicine, University of Chile. Santiago, Chile.

Institution where the study was executed: Clínica Las Condes. Santiago, Chile.

Fecha de recepción: 27 de noviembre de 2019

Fecha de aceptación: 12 de diciembre de 2019

ORCID

https://orcid.org/0000-0001-6355-1270

The authors declare no conflicts of interest.

There was no external funding for this project

Register: www.clinicaltrials.gov (NCT NCT03592056)

Corresponding author:

Julián Aliste, MD

julian.aliste@uchile.cl 
[0-0]; 0 [0-2]; 0 [0-2.5]; 0 [0-2], respectively. The median [IQR] consumption of LA boluses was 1.5 [0-7]. There were no postoperative morphine requirements. The most frequent side effect was Horner's syndrome. Conclusions: Continuous interscalene block with $0.04 \%$ levobupivacaine provides adequate analgesia for arthroscopic shoulder surgery but does not prevent hemidiaphragmatic paralysis at 24 hours under the conditions of this study.

\section{RESUMEN}

El bloqueo interescalénico representa el estándar analgésico para cirugía de hombro. No obstante, la incidencia de parálisis hemidiafragmática puede alcanzar $100 \%$ de los casos. Nuestra hipótesis es que infusiones con anestésicos locales más diluidos disminuirían la PHD 24 horas postbloqueo. Métodos: Serie prospectiva de pacientes sometidos a cirugía artroscópica electiva de hombro con bloqueo interescalénico continuo. Un bolo de $15 \mathrm{~mL}$ de lidocaína $1 \%$-levobupivacaína 0,5\% más infusión postoperatoria de levobupivacaína al 0,04\% a $8 \mathrm{ml} / \mathrm{h}$ más bolos a demanda de $5 \mathrm{~mL}$ con intervalo de 20 minutos hasta el alta. La excursión hemidiafragmática se evaluó con ultrasonido con transductor curvo 2-5 $\mathrm{MHz}$ en modo $\mathrm{M}$ en la región infracostal antes del bloqueo, en la unidad postanestésica y a las $24 \mathrm{~h}$, antes del alta. El outcome primario fue la presencia de parálisis hemidiafragmática 24 horas postbloqueo. Los resultados secundarios incluyeron dolor postoperatorio, total de bolos de rescate, requerimiento de opioides postoperatorios y efectos secundarios. Resultados: Treinta pacientes fueron reclutados y analizados. La incidencia de PHD a las 24 $\mathrm{h}$ fue $96,7 \%$. La mediana [RIC] de dolor en reposo (pacientes con inmovilizador de hombro) medido en escala numérica de 0 a 10 , a las 0,$5 ; 1 ; 3 ; 6 ; 12 ; 24$; 48; 72 horas fueron 0 [0-0]; 0 [0-0]; 0 [0-0]; 0 [0-0]; 0 [0-0]; 0 [0-2]; 0 [0-2.5]; 0 [0-2] respectivamente. La mediana [RIC] de consumo de bolos de rescate fue 1,5 [0-7]. No hubo pacientes con requerimientos de morfina postoperatoria. El efecto colateral más frecuente fue el síndrome de Horner. Conclusiones: El bloqueo interescalénico continuo con levobupivacaína 0,04\% proporciona analgesia postoperatoria adecuada, pero no evita la PHD a las $24 \mathrm{~h}$ en las condiciones de esta serie.

\section{Palabras clave:}

Bloqueo interescalénico continuo, parálisis hemidiafragmática, cirugía de hombro Términos MeSH: bloqueo plexo braquial

\section{Introduction}

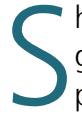
houlder surgery is among the most painful surgical interventions[1]. The interscalene brachial plexus block (ISB) represents the analgesic standard for these surgeries[1]. Despite its benefits, from the studies of Urmey et al., we know that ISB is associated with up to a $100 \%$ hemidiaphragmatic paralysis (HDP)[2] and a $27 \%$ decrease in forced vital capacity (FVC), and first-second forced expiratory volume (FEV 1)[3]. In general, these side effects are well tolerated in patients with normal respiratory reserve but prohibitive for those with restrictive or obstructive limitations.

The 2 millimeters distance between the brachial plexus and the phrenic nerve at the level at which the injection of local anesthetics (LA) is usually performed explains the high incidence of HDP with ISB[1].

Since the incorporation of ultrasound (US) into the regular practice of regional anesthesia, it has been possible to reduce the HDP incidence with more precise techniques for single doses ISB[1]. For example, $5 \mathrm{~mL}$ injections have shown incidences of just $20 \%[3]$. Also, the use of diluted concentrations of LA can reduce HDP incidence up to ranges between $21 \%[4]$ and $42 \%[5]$. However, extrapolation of these results to continuous interscalene brachial plexus blocks (CISB) is not possible and for the latter, up to a $100 \%$ incidence of HDP has been reported[6], and diaphragmatic and respiratory dysfunction has 
been demonstrated with infusions of $0.25 \%$ bupivacaine[7], $0.125 \%$ bupivacaine[8],[9] and with $0.2 \%$ ropivacaine[9],[10].

In clínica Las Condes, for more than a decade, the Department of Anesthesiology established the use of ultra-diluted concentrations of LA for all continuous regional blocks in search of a differential block trying to reduce the motor block and its potential complications. Thus, solutions of $0.04 \%$ levobupivacaine are continuously prepared by the pharmacy either for epidural or peripheral nerve infusions, for in- and outpatients.

In the present case series, we aimed to prospectively determine the incidence of HDP in patients who underwent arthroscopic shoulder surgery with CISB using $0.04 \%$ levobupivacaine for infusion as the pillar of postoperative analgesia.

\section{Methods}

After obtaining the approval of the Research Ethics Committee, this study was prospectively registered on July 19, 2018, before patient recruitment. After signing informed consent, 30 patients scheduled for arthroscopic shoulder surgery in whom postoperative analgesia based on CISB with levobupivacaine 0.04\% was planned were prospectively recruited. The inclusion criteria were: age between 18 and 80 years, classification of the American Society of Anesthesiologists (ASA) I - III, and body mass index between 20 and 35 $\mathrm{kg} / \mathrm{m}^{2}$. The exclusion criteria were: inability to consent to the study, preexisting pulmonary disease (obstructive or restrictive), coagulopathy, sepsis, liver or kidney failure, pregnancy, allergy to LA, previous surgery on shoulder to operate or cervical region to block, and condition of chronic pain requiring domiciliary opioids.

After admission to the operating room, patients were monitored according to the ASA standard, and an 18- or 20-gauge intravenous line was installed in the contralateral upper extremity contralateral to the surgical site. Then, a researcher evaluated the basal diaphragmatic excursion on the surgical side with a 2-5 MHz M-Turbo M-Turbo curvilinear US transducer in M mode (Sonosite, Bothell, Washington, USA). The liver and spleen served as acoustic windows on the right and left side, respectively. Patients were scanned at the subcostal border between the clavicular midline and the anterior axillary line with the US probe cranially directed[11]. The diaphragmatic excursion distance was evaluated in forced inspiration and recorded in millimeters (Figure 1).

Subsequently, with supplementary oxygen (mask $4 \mathrm{~L} / \mathrm{min}$ ) and after intravenous premedication (50 $\mu \mathrm{g}$ of fentanyl) that was administered to all patients, a US 6-15 MHz linear transducer Sonosite M-Turbo (Sonosite, Bothell, Washington, USA) was applied in a sterile fashion at the cervical level on the side to be blocked at the height of the cricoid cartilage to obtain a view of the hypoechoic structures, which represent the roots/trunks of the brachial plexus at this level[12]. Using an in-plane with the US puncture technique, with a lateral to medial direction, a papule was first made with $3 \mathrm{ml}$ of $1 \%$ lidocaine and then, using a continuous block kit Contiplex S Ultra 360 (B. Braun Medical AG, Melsungen, Germany), the block needle was inserted under the prevertebral fascia, advancing the tip into the fascial sheath that surrounds the plexus at this level, between the two most superficial hypoechoic structures and then threading a catheter 2-3 cm into this sheath to then proceed with trocar removal. Then a mixture of 15 milliliters of lidocaine $1 \%$-levobupivacaine $0.25 \%$ was deposited at this location, confirming its distribution under the US. Then, the catheter was secured to the patient's skin with an adhesive patch (Tegaderm 3M Health Care, St. Paul, $\mathrm{MN})$.

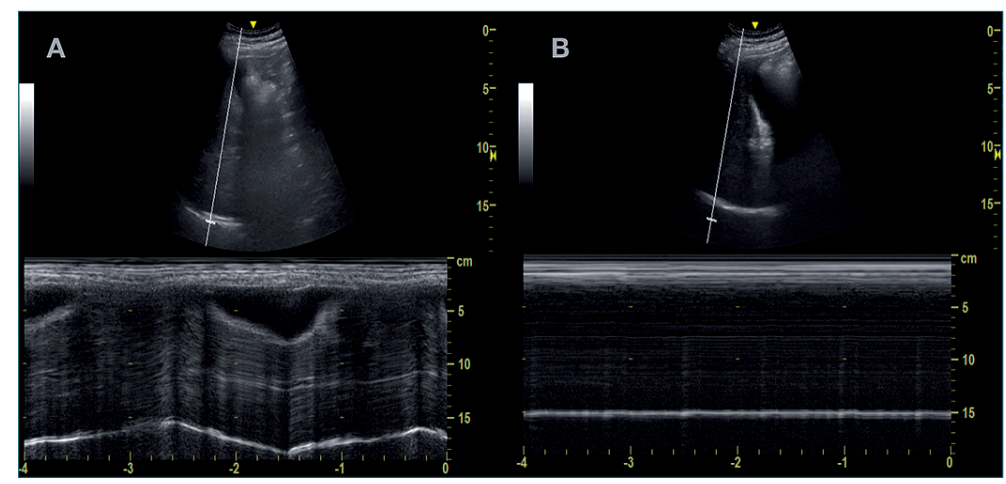

Figure 1. M-mode US for Diaphragm excursion measurement. A. Basal diaphragm excursion. Oscillating white line at $15 \mathrm{~cm}$ depth); B. Post block measurement. Static diaphragm. 
The intraoperative anesthetic technique was left at the discretion of the anesthetist involved in the care of the patient. However, the majority received general anesthesia induced with propofol, fentanyl, rocuronium and maintained with sevoflurane or desflurane with minimal alveolar concentration (MAC) 0.7-1.0, controlling the airway with endotracheal intubation or laryngeal mask.

All subjects were placed in a beach chair position for surgery. The surgical duration (interval between the insertion of the first trocar and skin closure) was recorded.

After surgery, a patient-controlled analgesia infuser (PCA) (Sapphire, QCore Medical Ltd, Netanya, Israel) with $0.04 \%$ levobupivacaine with an $8 \mathrm{~mL} / \mathrm{h}$ infusion plus $5 \mathrm{~mL}$ boluses with 20-minute lockout program was installed immediately after arriving at the post-anesthetic care unit (PACU) (Figure 2A). The adjuvant analgesia was indicated by the treating anesthesiologist and frequently consisted of around the clock IV or PO acetaminophen and a non-steroidalanti-inflammatories IV infusion (ketoprofen 300 mg or ketorolac 90 mg/24 h) plus q 20 min PRN IV 2 mg morphine in PACU and q 6-8 h PRN 50-100 mg of IV tramadol in the ward.

The patients were discharged after 24 hours postsurgery, replacing the PCA with a 250-mL elastomeric pump (Homepump C-series, Halyard Inc, Roswell, Georgia, USA) with a fixed infusion rate of $5 \mathrm{~mL} / \mathrm{h}$ (Figure 2B). After discharge, telephonic control was made through daily calls. All patients returned on the fourth postoperative day (POD) for catheter removal.

\section{Primary outcome}

During the first postoperative day, 24 hours af- ter the initial bolus of LA through the interscalene catheter, the diaphragmatic excursion was reassessed with the same methodology previously described. A diaphragm excursion decrease of less than $25 \%$ of the baseline value was considered within the possible normal variability. However, a drop between $25 \%$ to $75 \%$ was interpreted as partial diaphragmatic paralysis, and a difference greater than $75 \%$ was classified as total paralysis[13].

\section{Secondary outcomes}

Adverse events were recorded during block execution. (e.g., vascular puncture, toxic effects of LA, paresthesia).

Successful block was defined as the presence of sensory block to cold over the deltoid area and paresia/paralysis of the deltoid muscle, assessed by shoulder abduction.

At 30 minutes after arrival to PACU, diaphragmatic mobility was evaluated using the same method described and using the same definition of paresis and paralysis. Besides, pain scores were recorded at the same time using a numerical rating scale of 0-10 (0 = no pain; $10=$ worst pain imaginable) and cold-sensory blockade was tested in the deltoid region. Only pain at rest was assessed, given the indication of shoulder immobilization. Then, with the same numerical scale, pain at rest was evaluated at $1,3,6,12$, and 24 hours during hospitalization and by phone calls after discharge at POD 2 and 3. Also, PCA consumption, opioids requirements, and side effects associated with continuous blocks were registered. Finally, the use of intravenous dexamethasone was recorded as well, given its role in prolonging the effect of nerve blocks.

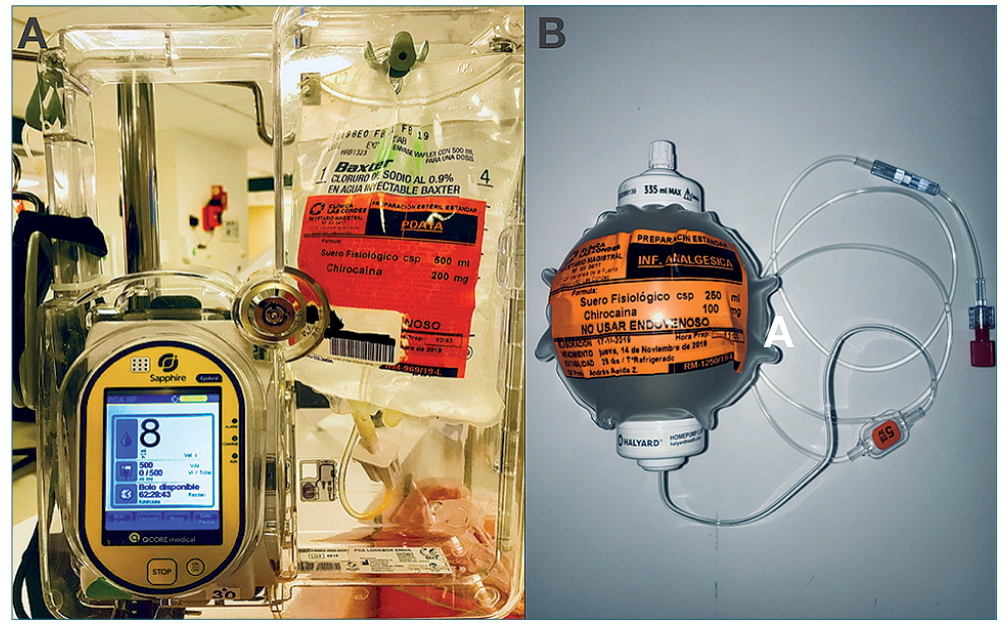

Figure 2. Infusion devices for continuous interscalene blocks. A. PCA pump with a $0.04 \%$ levobupivacaine solution at $8 \mathrm{~mL} / \mathrm{h}$ plus $5 \mathrm{~mL}$ on-demand bolus and a 20-minute lockout; B. $250 \mathrm{~mL}$ elastomeric pump with a $5 \mathrm{~mL} / \mathrm{h}$ fixedrate filled with $0.04 \%$ levobupivacaine. 


\section{Statistical analysis}

For the analysis of results, the Stata 15.0 program (StataCorp, 4905 Lakeway Dr, College Station, TX 77845, USA) was used for descriptive statistics, inferences, and association tests with contingency tables for possible risk factors of HDP. Depending on the type of variable and its distribution, the results are presented as means with standard deviation (SD), median with interquartile range and range [IQR] (Range) in addition to the use of counting and proportions with confidence interval $[\mathrm{CI}]$ when applicable.

\section{Results}

After a preliminary analysis of the first thirty subjects recruited, it was evident that the results regarding the primary outcome would not vary significantly under the methodology in the study, and it was decided to stop the recruitment. Thus, in this article, we present the results of these 30 cases, recruited between August 2018 and August 2019 (Table 1). All blocks were successful (sensory and motor), and in $96.7 \%$ [Cl $83-99 \%$ ] of cases, HDP (total $60.0 \%$ and partial $36.7 \%$ ) was demonstrated at $24 \mathrm{~h}$. However, the latter did not prevent patients from discharge.

In general, postoperative analgesia was adequate (Table 2), with a median [IQR] consumption PCA rescue boluses of LA during hospitalization of 1.5 [0-7] and no additional morphine requirements. In addition to HDP, the most common side effect was Horner's syndrome (Table 3). The use of intraoperative intravenous dexamethasone ( $60 \%$ of cases) was not correlated with differences in the presence of HDP (total or partial) at 24 hours after block commencement ( $p$ $=0.16$ ). On the third day after discharge, patients returned for catheter removal, reporting it as leaking in $23 \%$ of cases. No individual reported neurological deficits in his orthopedic control in POD 7.

\section{Discussion}

In this case series, we prospectively evaluated the incidence of HDP measured with US M-mode, 24 hours after the start of a CISB with an initial standard bolus but followed by a $0.04 \%$ levobupivacaine infusion, which has been the institutional standard for more than a decade, trying to determine if an infusion with this level of dilution prevents the presence of HDP (total or partial) prior to discharge in patients undergoing arthroscopic shoulder surgery.

\begin{tabular}{lc}
\multicolumn{2}{c}{ Table 1. Sample characteristics } \\
\hline Variable & Result \\
Age & $45(15)$ \\
Sex (M/F) & $25 / 15$ \\
BMI & $26.0(3.0)$ \\
ASA (I/II/III) & $20 / 10 / 0$ \\
Duration of surgery (min) & $70(31)$ \\
Basal diaphragm excursion (mm) & $52(12)$ \\
Intraoperative IV-dexamethasone use (\%) & $60 \%$ \\
\hline
\end{tabular}

Continuous variables expressed as mean (standard deviation); Categorical variables expressed as counting or percentage; ASA: Risk level American Association of Anesthesiology. BMl: body mass index $\mathrm{kg} / \mathrm{M}^{2}$.

\begin{tabular}{lc}
\hline & Table 2. Postoperative pain \\
\hline Static pain & Result (NRS) \\
$30 \mathrm{~min}$ & $0[0-0](0-8)$ \\
$60 \mathrm{~min}$ & $0[0-0](0-4)$ \\
$3 \mathrm{~h}$ & $0[0-0](0-4)$ \\
$6 \mathrm{~h}$ & $0[0-0](0-4)$ \\
$12 \mathrm{~h}$ & $0[0-0](0-5)$ \\
$24 \mathrm{~h}$ & $0[0-2](0-4)$ \\
$48 \mathrm{~h}$ & $0[0-2.5](0-8)$ \\
$72 \mathrm{~h}$ & $0[0-2](0-5)$
\end{tabular}

Ordinal variables expressed as median [interquartile range] (range); NRS: numeric rating scale from 0 to 10 .

Our findings suggest that at least with the protocol used, a low concentration of LA would not prevent the HDP within 24 hours of initiating a CISB, although it did not affect the quality of postoperative analgesia.

Our findings deserve further discussion. The HDP with ISB derives from two possible mechanisms: cranial ascent of the LA towards the nerve roots of C3 - C5 or its anterior migration from the interscalene groove towards the phrenic nerve, which at the usual level of injection is only 2 millimeters away[1]. In general, attempts to reduce HDP with single-dose ISB have sought to limit the injectate between the plexus and the middle scalene muscle[1] and decrease the drug mass, either in terms of volume or concentration[1]. However, none of these attempts has separately managed to lower the incidence of HDP by less than $20 \%$. 
Table 3. Block characteristics

\begin{tabular}{lc}
\hline Variable & Result \\
Successful block (\%) & 100 \\
HDP PACU (\% [Cl]) & $100[88-100]$ \\
Total (\%) & 86.7 \\
Partial (\%) & 13.3 \\
Normal (\%) & 0 \\
HDP 24 h (\% [Cl]) & $96.7[83-99]$ \\
Total (\%) & 60 \\
Partial (\%) & 36.7 \\
Normal (\%) & 3.3 \\
Horner's Syndrome (\%) & 23 \\
Block related complications (\%) & $0 / 0 / 0 / 0$ \\
(Vascular puncture/Paresthesia/Pneumothorax/LAST) & \\
Catheter related complications (\%) & $0 / 23 / 0$ \\
(Accidental dislodgement/Leak/infection) & \\
\hline
\end{tabular}

Categorical variables expressed as a percentage [confidence interval]; HDP: hemidiaphragmatic paralysis; PACU: post anesthetic recovery unit; Cl: confidence interval; LAST: local anesthetic systemic toxicity.

Studies of CISB and HDP analyzing various concentrations $(0.25 \%$ bupivacaine, $0.125 \%$ bupivacaine, $0.2 \%$ ropivacaine) have reported respiratory compromise in up to $100 \%$ of cases[6]. Most of these trials did not employ ultrasound either to guide the insertion of the catheter or to evaluate the diaphragmatic motility. Besides, large and concentrated initial boluses were employed, and the relationship between catheter tip and plexus was not confirmed (neither ultrasound nor neurostimulation). Also, the respiratory exams (spirometry, flowmetry, and chest $X$-ray) did not allow differentiating between total or partial paralysis[7]-[10].

A more recent study compared two positions of US-guided catheter tips (intrafascial and posterior-extrafascial) using an initial concentrated bolus $(20 \mathrm{~mL}$ $0.75 \%$ ropivacaine) followed by an infusion $(0.2 \%$ ropivacaine) at $4 \mathrm{~mL} / \mathrm{h}$ with on-demand $4 \mathrm{~mL}$ bolus of the same solution with a 30-minute lockout, evaluating HDP $24 \mathrm{~h}$ postoperatively[14]. The definition of HDP was excursion decrease higher than $75 \%$ compared to baseline (same as ours for total paralysis). Thus, Albrecht et al., placing the tip of the catheter outside and posteriorly to the fascial sheath surrounding the brachial plexus in the interscalene groove, managed to reduce the incidence of (total) paralysis from $47 \%$ to $15 \%$, but unfortunately, they do not describe the incidence of partial paralysis. Despite this, Albrecht et al. proved that the extrafascial location of the catheter did not impair the quality of analgesia. Thus, these findings open the possibility of ex- ploring our diluted concentration of levobupivacaine using alternative positions of the catheter tip.

Further discussion requires the initial bolus of LA and the use of adjuvants, overall if the diaphragmatic evaluation occurs 24 hours after their administration. In our series, we found $60 \%$ total paralysis and 37\% partial paralysis at 24 hours with an intrafascial catheter. In our series, $60 \%$ of the patients received intravenous dexamethasone as prophylaxis of nausea and vomiting, being this drug a proven nerve block adjuvant that prolongs the duration of both motor and sensory blockade either by intravenous or perineural route[15]. Although we found no association between the use of dexamethasone and the incidence of total or partial paralysis, we cannot rule out any influence on the presence of the latter at 24 hours post block.

Concerning diaphragmatic motility measurements, we recognize that in an ideal study protocol, at least one evaluation daily up to at least 24 post catheter removal is necessary to have a complete idea of HDP evolution in CISB. However, for design reasons, there were no post-discharge diaphragmatic evaluations, even at the time the patient returned for catheter removal.

Finally, we recognize the weakness of the quasiscientific design of this study, but since the search of diaphragm-sparing alternatives with brachial plexus blocks have become popular in recent literature and the use of very diluted LA solutions has represented the standard of care for CISB in our institution, we 
thought this methodology as adequate as a first attempt to scientifically test the possible benefits of this infusions in terms of diaphragm function and may even represent a proof of concept for future more scientific research protocol designs.

\section{Conclusions}

In short, the $0.04 \%$ levobupivacaine infusion in CISB provides adequate postoperative analgesia in arthroscopic shoulder surgery but does not prevent HDP (total or partial) 24 hours post-block in the setting of intrafascial catheters and with an initial bolus of con- centrated, long-lasting LA.

Therefore, future research on this matter should limit the initial bolus, as the single-dose studies have suggested, with extrafascial and lateral to the plexus injections, even using short-action LA such as lidocaine, or just as diluted as the solution to infuse. Regarding the continuous aspect of the block, keeping the infusion of diluted LA outside the plexus sheath represents an exciting option.

Furthermore, upcoming studies need to add a more systematic evaluation of diaphragmatic motility beyond 24 hours post-block and ideally supplemented with functional tests to correlate the level of paresis with its respiratory impact.

\section{References}

1. Tran DQ, Elgueta MF, Aliste J, et al. Diaphragm-sparing nerve blocks for shoulder surgery. Reg Anesth Pain Med 2017;42: 32-38. https://doi.org/10.1097/ AAP.0000000000000529

2. Urmey WF, Talts KH, Sharrock NE. One hundred percent incidence of hemidiaphragmatic paresis associated with interscalene brachial plexus anesthesia as diagnosed by ultrasonography. Anesth Analg 1991; 72:498-503. https:// doi.org/10.1213/00000539199104000-00014

3. Urmey WF, Gloeggler PJ. Pulmonary function changes during interscalene brachial plexus block: Effects of decreasing local anesthetic injection volume. Reg Anesth 1993; 18:244-9.

4. Thackeray EM, Swenson JD, Gertsch MC, et al. Diaphragm function after interscalene brachial plexus block: a doubleblind, randomized comparison of $0.25 \%$ and $0.125 \%$ bupivacaine. J Elbow Shoulder Surg. 2013;22:381-386. https://doi. org/10.1016/j.jse.2012.06.011

5. Wong AK, Keeney LG, Cheng L, et al. Effect of local anesthetic concentration $(0.2 \%$ vs $0.1 \%$ ropivacaine) on pulmonary function, and analgesia after ultrasound-guided interscalene brachial plexus block: a randomized controlled study. Pain Med. 2016; 17:2397-2403. https://doi. org/10.1093/pm/pnw057

6. Verelst $P$, van Zundert A. Respiratory impact of analgesic strategies for shoulder surgery. Reg Anesth PainMed 2013; 38: 50-3. https://doi.org/10.1097/ AAP.0b013e318272195d

7. Pere $P$, Pitkänen $M$, Rosenberg $\mathrm{PH}$, et al. Effect of continuous interscalene brachial plexus block on diaphragm motion and on ventilatory function. Acta Anaesth Scand 1992; 36:53-7. https://doi. org/10.1111/j.1399-6576.1992. tb03421.x

8. Pere $\mathrm{P}$. The effect of continuous interscalene brachial plexus block with $0.125 \%$ bupivacaine plus fentanyl on diaphragmatic motility and ventilatory function. Reg Anesth 1993; 19:93-7.

9. Choromanski DW, Patel PS, Frederick JM, et al. The effect of continuous interscalene brachial plexus block with $0.125 \%$ bupivacaine vs. $0.2 \%$ ropivacaine on pain relief, diaphragmatic motility, and ventilatory function. J
Clin Anesth 2015 Dec;27(8):61926. https://doi.org/10.1016/j. jclinane.2015.03.006

10. Borgeat A, Perschak H, Bird P, et al. Patient-controlled interscalene analgesia with ropivacaine $0.2 \%$ versus patient-controlled intravenous analgesia after major shoulder surgery: effects on diaphragmatic and respiratory function. Anesthesiology 2000; 92:102-8. https://doi. org/10.1097/00000542200001000-00020

11. Loyd T, Tang YM, Benson MD, et al. Diaphragmatic paralysis: the use of $\mathrm{M}$ mode ultrasound for diagnosis in adults. Spinal Cord. 2006; 44:505-508. https://doi. org/10.1038/sj.sc.3101889

12. Aliste J, Bravo D, Layera S, et al. A randomized comparison between interscalene and costoclavicular blocks for arthroscopic shoulder surgery. Reg Anesth Pain Med 2019; 44:472-477. https://doi.org/10.1136/rapm2018-100055

13. Renes SH, Spoormans HH, Gielen MJ, et al. Hemidiaphragmatic paresis can be avoided in ultrasound-guided supraclavicular brachial plexus block. Reg Anesth Pain Med 2009; 34: 595-9. https://doi.org/10.1097/ 
AAP.0b013e3181bfbd83

14. Albrecht E, Bathory I, Fournier $\mathrm{N}$, et al. Reduced hemidiaphragmatic paresis with extrafascial compared with conventional intrafascial tip placement for continuous interscalene brachial plexus block: a randomized, controlled, double-blind trial. $\mathrm{Br}$ J Anaesth 2017118 (4): 58692. https://doi.org/10.1093/bja/ aex050

15. Kirkham KR, Jacot-Guillarmod A, Albrecht E. Optimal dose of perineural dexamethasone to prolong analgesia after brachial plexus blockade: a systematic review and meta-analysis. Anesth Analg 2018; 16:270-

9. https://doi.org/10.1213/ ANE.0000000000002488 